\title{
Measure based metrics for aggregated data
}

\author{
V.J. Rayward-Smith \\ School of Computing Sciences, University of East Anglia, Norwich, NR4 7TJ, UK \\ E-mail:vjrs@uea.ac.uk
}

\begin{abstract}
Aggregated data arises commonly from surveys and censuses where groups of individuals are studied as coherent entities. The aggregated data can take many forms including sets, intervals, distributions and histograms. The data analyst needs to measure the similarity between such aggregated data items and a range of metrics are reported in the literature to achieve this (e.g. the Jaccard metric for sets and the Wasserstein metric for histograms). In this paper, a unifying theory based on measure theory is developed that establishes not only that known metrics are essentially similar but also suggests new metrics.
\end{abstract}

Keywords: Measure theory, metric space, similarity, clustering, symbolic data, aggregated data

\section{Introduction}

Large data sets concerning individual entities found in medical, census or financial databases, for example, are often too large and/or too sensitive to be released to a wider community. To facilitate analysis of such data, it is common practice to aggregate the data based on individuals into data based upon groups of individuals. For example, with census data, data might be generated and analysed that describes geographically based communities. This can not only protect the individual but also be used as a means of comparing communities and thereby targeting strategic government funding. For medical data, aggregated data might be based on hospitals or health authorities and comparisons between them can then be made. Once the data has been aggregated, not only is it more manageable but it can often be safely released to a wide community, perhaps even to the general public.

Aggregated data, often referred to as symbolic data [2,3], usually have a markedly different structure from that of an individual. An entry for an individual might have a field describing the individual's age. The corresponding data for a group of individuals might be a set of ages, an interval of ages or a histogram describing the distribution of ages within the group.

The analyst needs to compare one group with another and thus needs techniques to measure the similarity between aggregated data. To measure similarities between items, it is common to seek a metric (or perhaps a pseudometric). In this paper, metrics and pseudometrics are defined over various types of aggregated data. These measures can then be combined to get an overall measure of similarity between the aggregated groups. Defining such measures correctly is important because it can affect policy and investment, internationally, nationally and locally, by companies, organisations and by governments.

In this paper, metrics and pseudometrics for aggregated data are studied. By introducing some simple measure theory, such metrics and pseudometrics are seen to have much in common; they are all special cases of one of two (pseudo)metrics defined in terms of a measure over an algebra. Section 2 introduces the measure theory required and Section 3 defines a metric space and explains how metrics and pseudometrics can be derived for an algebra over which a measure is defined. Then, in Section 4 , 
the theory that has been developed is applied to generate metrics and pseudometrics over sets, intervals and histograms. Throughout, care is taken to distinguish between categorical data that is nominal and that which is ordinal, as well as distinguishing between categorical and numeric data. The last section of the paper presents conclusions and some suggestions for further research.

Metrics are important in the analysis of unaggregated data, especially in clustering applications (see, e.g. [17]). Their use is dicussed further in [19] together with various metric based maesures for cluster qulaity. A scalable, metric based algorithm is described in [9]. Studying metrics for aggregated data is also not new. There is a large and growing corpus of work in this area both of a theoretical and of an applied nature, see [2-4,6-8,10-12,15], several of which include case studies relating to the analysis of census data. This paper provides a unifying theory for many existing metrics used in these articles and also produces some new metrics.

\section{Finitely additive measures}

Let $S$ be a set and let $\Sigma$ be a non-empty set of subsets of $S$ that is closed under complement and union. Thus, if $A$ is in $\Sigma$ then so is the complement of $A, A^{\prime}=S \backslash A$. Similarly if $A, B$ are in $\Sigma$ then $A \cup B$ is also in $\Sigma$. Providing these properties are satisfied, $(S, \Sigma)$ is called an algebra. By applying de Morgan's law, any algebra, $(S, \Sigma)$, will also be closed under intersection.

A finitely additive measure, $\mu$, on an algebra, $(S, \Sigma)$, is a function

$$
\mu: \Sigma \rightarrow R \cup\{\infty\}
$$

such that

1. $\mu(A) \geqslant 0$ for all $A \in \Sigma$,

2. $\mu(A)=0$ if $A=\emptyset$,

3. If $A, B$ are disjoint sets in $\Sigma$ then

$$
\mu(A \cup B)=\mu(A)+\mu(B) .
$$

A finitely additive measure is a relaxed form of a measure. A measure is defined on a $\sigma$-algebra, which is an algebra that is also closed under the union of a countable number of sets, rather than just a finite number of sets, see for example, [1,13]. A measure then has all the properties of a finitely additive measure but also satisfies the additional propert that if $A_{1}, A_{2}, \ldots$ is a countably infinite sequence of disjoint sets in the $\sigma$-algebra then

$$
\mu\left(\bigcup A_{i}\right)=\sum \mu\left(A_{i}\right)
$$

If $\mu(A)$ is finite for all $A \in \Sigma$, a finitely additive measure is called finite and all of the example measures used in this paper are indeed finite.

A finitely additive measure, $\mu$, will be called strong if $\mu(A)=0 \Rightarrow A=\emptyset$. Not all finitely additive measures discussed here are strong but, when they are, a metric can be constructed rather than just a pseudometric.

For any sets $A, B \in \Sigma$, the sets $A \backslash B, B \backslash A$ and $A \cap B$ are mutually disjoint. Thus, the following can be deduced. 


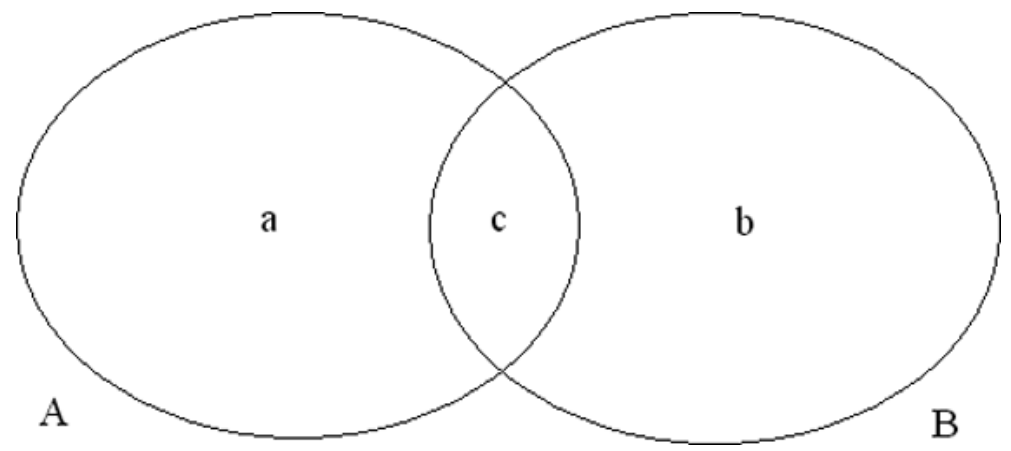

Fig. 1. Two intersecting sets.

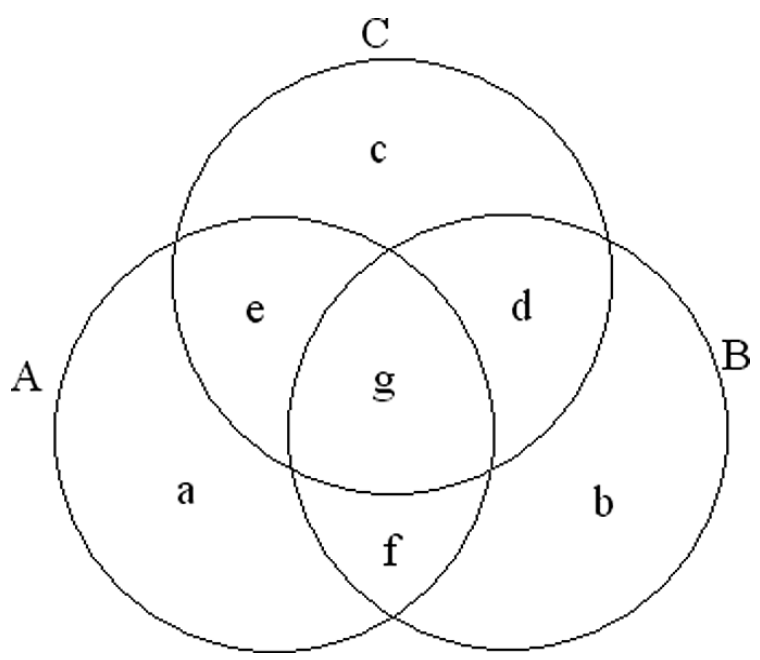

Fig. 2. Three intersecting sets.

Proposition 1 For any finitely additive measure, $\mu$, on the algebra $(S, \Sigma)$ and for any sets, $A, B \in \Sigma$, if $a=\mu(A \backslash B), b=\mu(B \backslash A)$ and $c=\mu(A \cap B)$, as in Fig. 1, then

$$
\begin{aligned}
\mu(A) & =\mu(A \backslash B)+\mu(A \cap B)=a+c, \\
\mu(B) & =\mu(B \backslash A)+\mu(A \cap B)=b+c \text { and } \\
\mu(A \cup B) & =\mu(A \backslash B)+\mu(B \backslash A)+\mu(A \cap B)=a+b+c .
\end{aligned}
$$

Similarly,

Proposition 2 For any three sets, $A, B, C$ in $\Sigma$, if $a=\mu(A \backslash B \backslash C), b=\mu(B \backslash A \backslash C), c=$ $\mu(C \backslash A \backslash B), d=\mu((B \cap C) \backslash A), e=\mu((C \cap A) \backslash B), f=\mu((A \cap B) \backslash C)$ and $g=\mu(A \cap B \cap C)$, as in Fig. 2, then

$$
\begin{aligned}
& \mu(A)=a+e+f+g, \\
& \mu(B)=b+d+f+g, \\
& \mu(C)=c+d+e+g,
\end{aligned}
$$




$$
\begin{aligned}
& \mu(A \cup B)=a+b+d+e+f+g, \\
& \mu(B \cup C)=b+c+d+e+f+g \text { and } \\
& \mu(A \cup C)=a+c+d+e+f+g .
\end{aligned}
$$

These two propositions are key to proving the following results on metrics and to understanding this paper.

\section{Metrics}

To be a metric on $\Sigma$, a distance function $\delta: \Sigma \times \Sigma \rightarrow R_{0}^{+}$must satisfy:

1. $\delta(A, B) \geqslant 0$,

2. $\delta(A, B)=0$ if and only if $A=B$,

3. $\delta$ is symmetric, i.e. $\delta(A, B)=\delta(B, A)$ for all $A, B \in \Sigma$, and

4. $\delta$ satisfies the triangle inequality, i.e. $\delta(A, B)+\delta(B, C) \geqslant \delta(A, C)$ for all $A, B, C \in \Sigma$.

$(\Sigma, \delta)$ is then called a metric space.

Metrics are used to define the difference between objects in the set $\Sigma$ and are widely used both to compare objects and within clustering algorithms, see e.g. [17].

If $\delta$ satisfies all the conditions of being a metric, except that $\delta(A, B)=0$ can occur when $A \neq B$, then $\delta$ is called a pseudometric. Clearly, any pseudometric will infer a metric on the equivalence classes of $\Sigma$ defined by the equivalence relation $A \sim B$ iff $\delta(A, B)=0$.

Given a finitely additive measure, $\mu$, on an algebra, $(S, \Sigma)$, the distance function, $\delta_{1}: \Sigma \times \Sigma \rightarrow R_{0}^{+}$ is defined by

$$
\delta_{1}(A, B)=\mu(A \cup B)-\mu(A \cap B)=\mu(A \backslash B)+\mu(B \backslash A) .
$$

Then,

1. $\delta_{1}(A, B) \geqslant 0$ since, by Proposition $1, \mu(A \cup B)-\mu(A \cap B)=\mu(A \backslash B)+\mu(B \backslash A)$, which must be $\geqslant 0$,

2. $\delta_{1}(A, B)=0$ if $A=B$ since then $\mu(A \cup B)-\mu(A \cap B)=\mu(A)-\mu(A)=0$,

3. if $\delta_{1}(A, B)=0$ then $\mu(A \backslash B)+\mu(B \backslash A)=0$ and thus both $\mu(A \backslash B)=0$ and $\mu(B \backslash A)=0$. If $\mu$ is a strong measure then it follows that $A \backslash B=\emptyset$ and $B \backslash A=\emptyset$ and hence, $A=B$,

4. $\delta_{1}(A, B)=\delta_{1}(B, A)$ by the symmetry of the definition, and

5. using the notation of Fig. 2, for any sets, $A, B, C \in \Sigma$,

$$
\begin{aligned}
\delta_{1}(A, B)+\delta_{1}(B, C) & =a+e+b+d+b+f+c+e \\
& \geqslant a+f+c+d \\
& =\delta_{1}(A, C)
\end{aligned}
$$

By the results listed above for $\delta_{1}$, the following result is established.

Theorem 1. Given a finitely additive measure, $\mu$, on an algebra, $(S, \Sigma)$, a pseudometric, $\delta_{1}: \Sigma \times \Sigma \rightarrow$ $R_{0}^{+}$can be defined by

$$
\delta_{1}(A, B)=\mu(A \cup B)-\mu(A \cap B) .
$$

Moreover, if $\mu$ is a strong measure then $\delta_{1}$ is a metric. 
An alternative distance measure, $\delta_{2}: \Sigma \times \Sigma \rightarrow R_{0}^{+}$, is defined by

$$
\delta_{2}(A, B)=\left\{\begin{array}{lr}
0 & \text { if } A=B=\emptyset, \\
1-\frac{\mu(A \cap B)}{\mu(A \cup B)} & \text { otherwise. }
\end{array}\right.
$$

Then $0 \leqslant \delta_{2}(A, B) \leqslant 1$ since $0 \leqslant \mu(A \cap B) \leqslant \mu(A \cup B)$. Clearly

1. $\delta_{2}(A, A)=0$,

2. $\delta_{2}(A, B)=0$ implies $\mu(A \cap B)=\mu(A \cup B)$ and hence $\mu(A \backslash B)=\mu(B \backslash A)=0$. Thus, if $\mu$ is strong, this implies $A \backslash B=B \backslash A=\emptyset$ and hence $A=B$,

3. $\delta_{2}(A, B)=\delta_{2}(B, A)$.

The triangle inequality is also satisfied as shown in the lemma below.

Lemma 1. For any subsets, $A, B, C$ of $\Sigma$,

$$
\delta_{2}(A, B)+\delta_{2}(B, C) \geqslant \delta_{2}(A, C) .
$$

Proof: Referring to Fig. 2 and assuming $S=a+b+c+d+e+f+g$ and $S-c \neq 0$ then

$$
\delta_{2}(A, B)=1-\frac{f+g}{S-c} .
$$

Similarly,

$$
\delta_{2}(B, C)=1-\frac{d+g}{S-a}
$$

provided $S-a \neq 0$ and

$$
\delta_{2}(A, C)=1-\frac{e+g}{S-b}
$$

provided $S-b \neq 0$.

Then, provided $(S-a)(S-b)(S-c) \neq 0$,

$$
\begin{aligned}
& \delta_{2}(A, B)+\delta_{2}(B, C)-\delta_{2}(A, C) \\
& =1-\frac{f+g}{S-c}+1-\frac{d+g}{S-a}-1+\frac{e+g}{S-b} \\
& =1-\frac{(f+g)(S-a)(S-b)+(d+g)(S-b)(S-c)-(e+g)(S-a)(S-c)}{(S-a)(S-b)(S-c)} .
\end{aligned}
$$

After some tedious algebra, the numerator of this expression evaluates to a sequence of terms that are all non-negative.

On the assumption that $(S-a)(S-b)(S-c) \neq 0$, the denominator is also positive, so we can deduce that $\delta_{2}(A, B)+\delta_{2}(B, C)-\delta_{2}(A, C) \geqslant 0$ and thus the triangle inequality holds.

The above argument relies on the assumption that $(S-a)(S-b)(S-c) \neq 0$. Now $(S-a)(S-$ $b)(S-c)=0$ iff one or more of $(S-a),(S-b)$ or $(S-c)$ is zero iff at least two of the sets are empty. If $A=B=C=\emptyset$ then $\delta_{2}(A, B)=\delta_{2}(B, C)=\delta_{2}(A, C)=0$ and the triangle inequality holds. If $A=B=\emptyset$ and $C \neq \emptyset$ then $\delta_{2}(A, C)=\delta_{2}(B, C)=1$ and $\delta_{2}(A, B)=0$ so the triangle inequality holds. The argument for the remaining cases are similar. The proof is thereby completed.

Hence, the following can be deduced. 
Theorem 2. The distance function $\delta_{2}: \Sigma \times \Sigma \rightarrow R_{0}^{+}$defined by

$$
\delta_{2}=\left\{\begin{array}{lr}
0 & \text { if } A=B=\emptyset, \\
1-\frac{\mu(A \cap B)}{\mu(A \cup B)} & \text { otherwise }
\end{array}\right.
$$

is a pseudometric on $\Sigma$ and, moreover, if $\mu$ is a strong measure then $\delta_{2}$ is a metric.

Proof: The pseudometric result follows from the lemma above and the preceeding observations. If $\mu$ is strong then

$$
\begin{aligned}
\delta_{2}(A, B)=0 & \Rightarrow \mu(A \cup B)=\mu(A \cap B) \\
& \Rightarrow \mu(A \backslash B)=\mu(B \backslash A)=0 \\
& \Rightarrow A \backslash B=B \backslash A=\emptyset \\
& \Rightarrow A=B
\end{aligned}
$$

and hence $\delta_{2}$ is a metric.

\section{Applications to aggregated data}

In this section, we consider examples of aggregated data and show how measures can be defined and metrics deduced.

\subsection{Finite sets}

One of the most common examples of aggregated data is a set. Say a database has a field, F, with values that are categorical. Now, consider aggregating data from field $\mathrm{F}$ from $n$ records, $r_{1}, r_{2}, \ldots, r_{n}$. The result may be a set of values taken by field $\mathrm{F}$ for these $n$ records.

Given a finite set, $S$, the cardinality function, $\mu_{c}: 2^{S} \rightarrow Z \subset R$ is defined by

$$
\mu_{c}(A)=|A| .
$$

Clearly this is a finitely additive measure on the algebra $2^{S}$ and, moreover, it is a strong measure. Hence the following.

Corollary 1 If $S$ is a finite set then the following are both metrics on $2^{S}$ :

$$
\begin{aligned}
& \text { 1. } \delta_{1}^{c}(A, B)=|A \cup B|-|A \cap B|=|A \backslash B|+|B \backslash A|, \\
& \text { 2. } \delta_{2}^{c}=\left\{\begin{array}{lc}
0 & \text { if } A=B=\emptyset, \\
1-\frac{|A \cap B|}{|A \cup B|}=\frac{|A \backslash B|+|B \backslash A|}{|A \cup B|}, & \text { otherwise. }
\end{array}\right.
\end{aligned}
$$

The first metric is the usual metric for sets, the second is known as the Jaccard metric [16]. Both have been used to cluster sets; for example, in [20], sets of support for partial classification rules were clustered using $\delta_{2}^{c}$ in order to identify rules that were similar semantically and thereby to gain a better understanding of the data. 


\subsection{Finite sets of ordinals}

Field values may be ordinal; if the values lie in a finite ordinal set, $S$, then there will be a function $\rho: S \rightarrow R^{+}$. This may be a simple ranking function whereby the $i^{t h}$ element of the set is assigned $i$ or it may be a more sophisticated assignment. For example, DEGREECLASS may contain values from 1st, 2(i), 2(ii), 3rd, Pass, Fail and a simple ranking would assign these values to integers 1, 2, 3, 4, 5 and 6, respectively. An alternative assignment that perhaps better reflects their relative merit would be to assign each classification to the average of the marks in the span. Using a UK marking scheme, this might result in an assignment of $85,65,55,45,37,17.5$, respectively.

Let $S$ be a finite set of ordinal data and $\rho: S \rightarrow R^{+}$be an injection. Then $\left(S, 2^{S}\right)$ is an algebra and the rank measure induced by $\rho$ is

$$
\mu_{\rho}(A)=\sum_{x \in A} \rho(x)
$$

Then $\mu_{\rho}$ is a finite measure and, since $\rho>0, \mu_{\rho}(A)=0$ only when $A=\emptyset$. Thus $\mu_{\rho}$ is also a strong measure and hence the following result.

Corollary 2 If $S$ is a finite ordinal set and $\rho: S \rightarrow R^{+}$is an injection then the following are both metrics on $2^{S}$ :

1. $\quad \delta_{1}^{\rho}(A, B)=\sum_{x \in A \cup B} \rho(x)-\sum_{x \in A \cap B} \rho(x)=\sum_{x \in A \backslash B} \rho(x)+\sum_{x \in B \backslash A} \rho(x)$,

2. $\delta_{2}^{\rho}(A, B)=\left\{\begin{array}{lr}0 & \text { if } A=B=\emptyset, \\ 1-\frac{\sum_{x \in A \cap B} \rho(x)}{\sum_{x \in A \cup B} \rho(x)} & \text { otherwise. }\end{array}\right.$

which is equivalent to

$$
\delta_{2}^{\rho}(A, B)=\left\{\begin{array}{lr}
0 & \text { if } A=B=\emptyset, \\
\frac{\sum_{x \in A \backslash B} \rho(x)+\sum_{x \in B \backslash A} \rho(x)}{\sum_{x \in A \cup B} \rho(x)} & \text { otherwise. }
\end{array}\right.
$$

\subsection{Intervals}

Let $S$ be the interval of the real line, $[a, b]$ say, and let $\Sigma$ denote all the finite sets of subintervals of $[a, b]$. A subinterval is either the empty set or may be open, closed or half open, i.e. of the form

1. $(c, d)=\{x \mid a \leqslant c<x<d \leqslant b\}$,

2. $[c, d)=\{x \mid a \leqslant c \leqslant x<d \leqslant b\}$,

3. $(c, d]=\{x \mid a \leqslant c<x \leqslant d \leqslant b\}$ or

4. $[c, d]=\{x \mid a \leqslant c \leqslant x \leqslant d \leqslant b\}$.

Then $(S, \Sigma)$ is an algebra.

The width measure is defined on any interval, $I=(c, d),[c, d),(c, d]$, or $[c, d]$ by

$$
\mu_{w}(I)=d-c .
$$

Two intervals are said to be disjoint if their union is not itself an interval. A union of a finite number of intervals can clearly be expressed uniquely as a union of pairwise disjoint intervals. If $A$ is an element 
of $\Sigma$, i.e. a set of intervals in $[a, b]$, then $\hat{A}$ will represent the corresponding set of pairwise disjoint intervals.

The function $\mu_{w}$ can then be extended to elements of $\Sigma$ in the obvious way by defining

1. $\mu_{w}(\Phi)=0$, and

2. for any nonempty set of intervals $A \in \Sigma, \mu_{w}(A)$ is the sum of the widths of the pairwise disjoint intervals in $\hat{A}$, i.e.

$$
\mu_{w}(A)=\sum_{I \in \hat{A}} \mu_{w}(I)
$$

This measure is finite since for any set of intervals, $A$ in $[a, b], \mu_{w}(A) \leqslant b-a$. However, since $\mu_{w}\{[x, x]\}=0$ for any $x \in[a, b]$, it is not a strong measure.

Corollary 3 If $A, B \in \Sigma$ denote finite sets of intervals in $[a, b]$ then the following are both pseudometrics on $\Sigma$ :

1. $\delta_{1}^{w}(A, B)=\mu_{w}(A \cup B)-\mu_{w}(A \cap B)$,
2. $\delta_{2}^{w}(A, B)=\left\{\begin{array}{lr}0 & \text { if } A=B=\emptyset, \\ 1-\frac{\mu_{w}(A \cap B)}{\mu_{w}(A \cup B)} & \text { otherwise. }\end{array}\right.$

\subsection{Regions of the Euclidean Plain}

Let $S$ be a finitely bounded, closed region of $R^{2} . S$ has a finite perimeter and contains all the points on the perimeter and in the region bounded by that perimeter. Let $B$ denote all finitely bounded regions within $S$. An element of $B$ is a subspace of $S$ and will be contained by a perimeter but may or may not contain points on that perimeter, i.e. it may be be closed or open. Now, let $\Sigma$ denote the closure of $B$ under union and complement so that $\Sigma$ is an algebra.

Every $A \in \Sigma$ has a finite area less than or equal to the finite area of $S$. The area of $A$, denoted by $\mu_{a}(A)$ provides a finitely additive measure on $(S, \Sigma)$. It is not a strong measure since if $A$ is an open region in $S$, i.e. does not contain its boundary, whilst $\bar{A}$ is the corresponding closed region, i.e. $A$ together with its boundary, then $\mu_{a}(A)=\mu_{a}(\bar{A})$ although $A \neq \bar{A}$.

Area measures are of particular interest to analysts of aggregated data when applied to distributions and to histograms.

Let $I=[a, b]$ be an interval and let $c$ be a positive real. Then $\mathcal{F}_{a, b, c}$ denotes the set of continuous functions on I bounded so that

$$
\text { if } f \in \mathcal{F}_{a, b, c} \text { then } 0 \leqslant f(x) \leqslant c \text { for all } x \in I .
$$

Any $f \in \mathcal{F}_{a, b, c}$ defines a region, $X_{f}$, bounded by the perimeter comprising four lines

1. $\{(x, y) \mid x=a, 0 \leqslant y \leqslant f(a)\}$,

2. $\{(x, y) \mid x=b, 0 \leqslant y \leqslant f(b)\}$,

3. $\{(x, y) \mid a \leqslant x \leqslant b, y=0\}$,

4. $\{(x, y) \mid a \leqslant x \leqslant b, y=f(x)\}$

as in Fig. 3. 


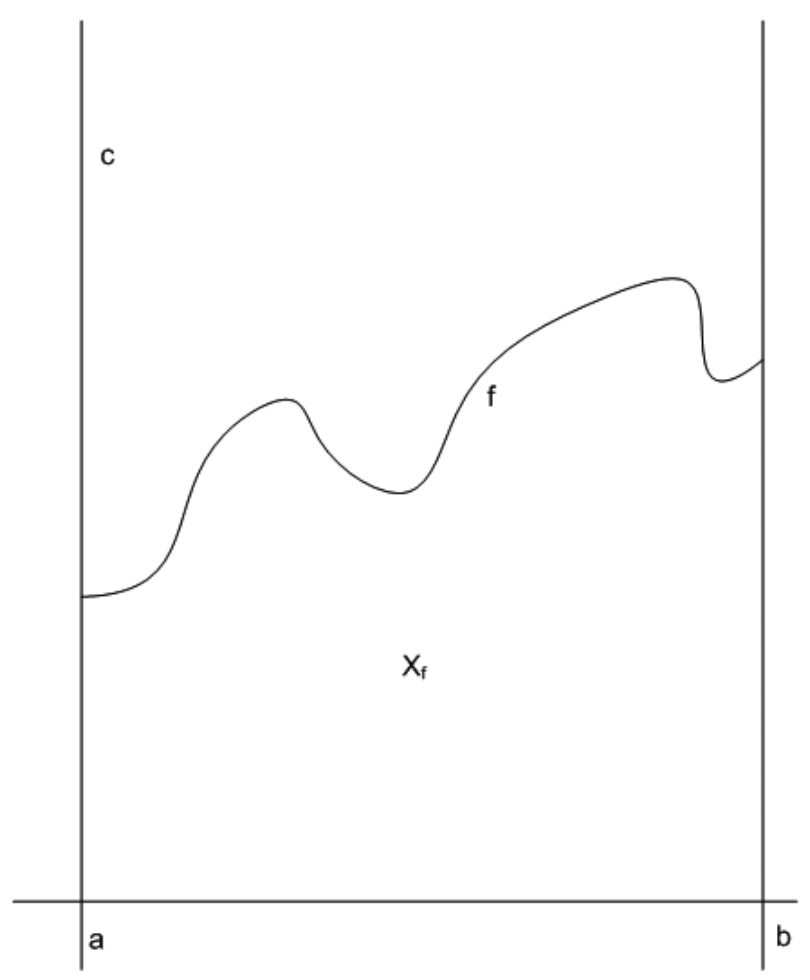

Fig. 3. The region $X_{f}$.

The area of the region $X_{f}$ is then

$$
\int_{x=a}^{b} f(x) d x
$$

By applying the area pseudometric, the following can be deduced.

Corollary 4 The following are metrics on elements of $\mathcal{F}_{a, b, c}$.

$$
\begin{aligned}
& \text { 1. } \int_{x=a}^{b}|f(x)-g(x)|=\int_{x=a}^{b}(\max (f(x), g(x))-\min (f(x), g(x))), \\
& \text { 2. } \quad 1-\frac{\int_{x=a}^{b} \min (f(x), g(x))}{\int_{x=a}^{b} \max (f(x), g(x))} \text { providing } f, g \text { are not both everywhere } 0 .
\end{aligned}
$$

Proof:

1. The integral simply gives the value of $\mu_{a}\left(X_{f} \backslash X_{g}\right)+\mu_{a}\left(X_{g} \backslash X_{f}\right)$ and hence, by Theorem 1, is a pseudometric. However,

$$
\int_{x=a}^{b}|f(x)-g(x)|=0 \Rightarrow f(x)=g(x) \forall x \in[a, b], \text { i.e. } f=g \text { on } I
$$

and hence the integral is also a metric.

2. This follows from Theorem 2 using a similar argument. 


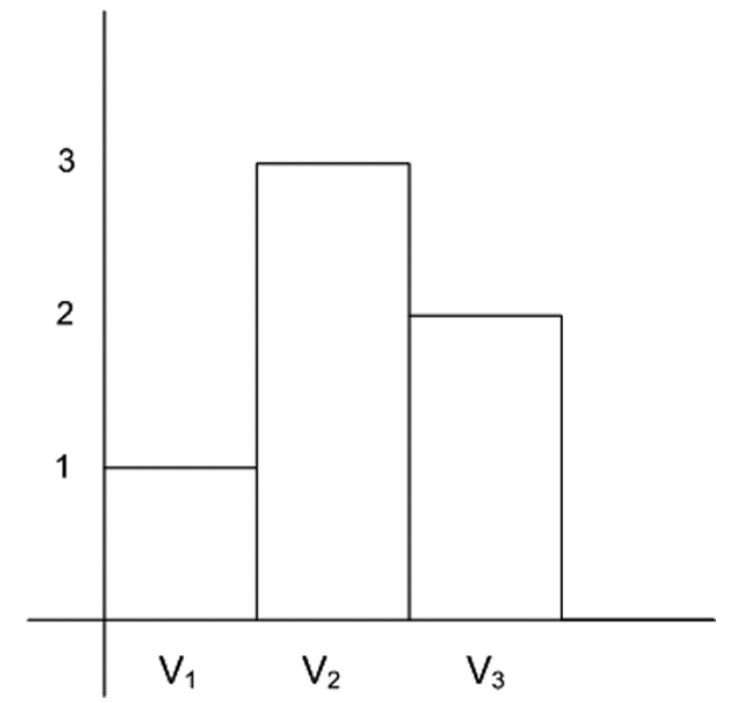

Fig. 4. Representing a simple histogram on $\left\{V_{1}, V_{2}, V_{3}\right\}$.

\subsection{Histograms}

Let $\mathrm{F}$ be a field of a database of $n$ records that has been aggregated to produce a histogram. The field, F, may be nominal, ordinal or real-valued. Each case will be considered separately.

\subsubsection{Histograms over nominal sets}

In the nominal case, the possible values in $\mathrm{F}$ will be finite in number. Let $V$ denote the set of values that are enumerated as $\left\{v_{1}, v_{2}, \ldots, v_{m}\right\}$. A histogram for $\mathrm{F}, H$, over $V$ is determined by

1. a partition of $V$ into disjoint, nonempty, subsets, $V_{1}, V_{2}, \ldots, V_{k}, k \leqslant m$ and,

2. for each $1 \leqslant i \leqslant k$, a count, $c^{H}\left(V_{i}\right) \in Z_{0}^{+}$, of the number of occurrences of elements in $V_{i}$ that occur in field $\mathrm{F}$ of the database.

Commonly, but not necessarily, each $V_{i}$ is a singleton set. If $H$ is such a histogram and $V_{i}=\left\{v_{i}\right\}$ then $c^{H}\left(\left\{v_{i}\right\}\right)$ may be expressed as $c^{H}\left(v_{i}\right)$.

Note that in all cases

$$
n=\sum_{k=1}^{m} c^{H}\left(V_{i}\right)
$$

is the number of elements in the underlying database and this will be called the base number of the histogram.

There are two ways of representing a histogram, $H$ over $V$, in $R^{2}$. The first provides equal width partitions of the x-axis for each of $V_{1}, V_{2}, \ldots, V_{k}$ and comprises a series of rectangles $R_{i}, 1 \leqslant i \leqslant k$, where $R_{i}=[i-1, i) \times\left(0, c^{H}\left(V_{i}\right)\right)$.

Thus, if $\mathrm{F}$ comprises the values $1,2,2,3,4,4$ and $V_{1}=\{1\}, V_{2}=\{2,3\}, V_{3}=\{4\}$, the histogram can be represented as in Fig. 4.

However, it is also common to label the x-axis with the elements of $V_{1}$, followed by the elements of $V_{2}$, etc. Then a rectangle, $R_{i}$, is drawn for each $V_{i}$ of width $\left|V_{i}\right|$ and height $\frac{c^{H}\left(V_{i}\right)}{\left|V_{i}\right|}$. For the above example and using the listing of $V$ to be $v_{1}, v_{2}, v_{3}, v_{4}$, this results in the histogram of Fig. 5 . 


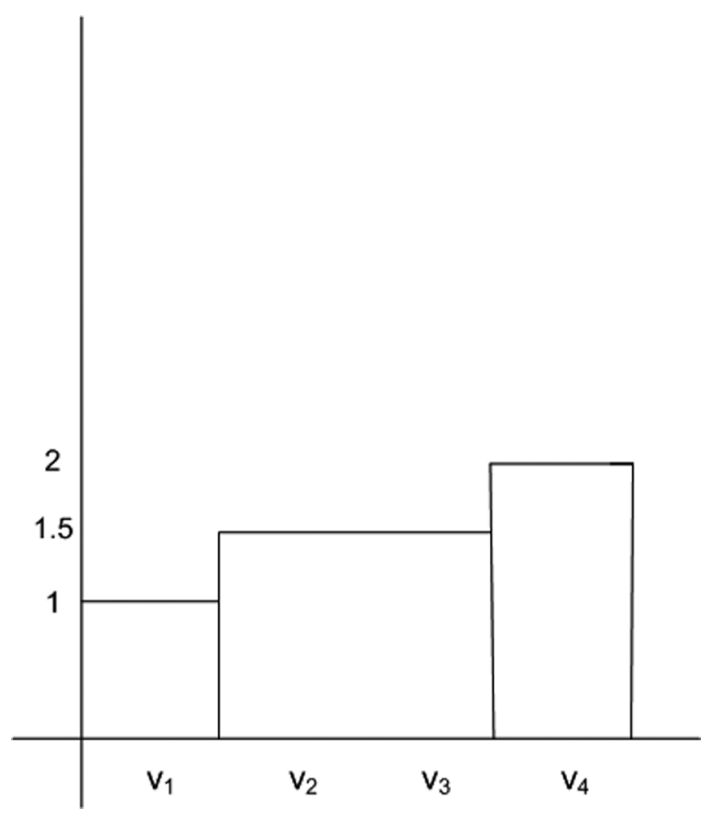

Fig. 5. Representing a simple histogram on $\left\{v_{1}, v_{2}, v_{3}, v_{4}\right\}$.

Note that, with either representation, the total area of the representation of the histogram is its base number.

Assume now that $H_{1}$ and $H_{2}$ are two distinct histograms over $V_{1}$ and $V_{2}$, respectively, where $\left|V_{1}\right|=m_{1}$ and $\left|V_{2}\right|=m_{2}$. These two histograms are to be compared.

If $V_{1} \neq V_{2}$, then set $V=V_{1} \cup V_{2}$ and regard each of $H_{1}$ and $H_{2}$ to be a histogram over $V$ setting $c^{H_{1}}\left(V \backslash V_{1}\right)=0$ if $V \backslash V_{1} \neq \emptyset$ and, likewise, $c^{H_{2}}\left(V \backslash V_{2}\right)=0$ if $V \backslash V_{2} \neq \emptyset$. Let $n_{1}$ denote the base number of $H_{1}$ and $n_{2}$ denote the base number of $H_{2}$. The histograms are then scaled by setting $n=l c m\left(n_{1}, n_{2}\right)$, and multiplying $c^{H_{1}}\left(V_{i}\right)$ by $n / n_{1}$ and $c^{H_{2}}\left(V_{i}\right)$ by $n / n_{2}$. The two histograms are then over the same set (although not necessarily using the same partition of this set) and have the same base number, $n$.

For example, consider two histograms, $H_{1}$ and $H_{2}$, where

1. $H_{1}$ is defined over $\{1,2,3,4\}$ and partitions this set into the three subsets $V_{11}=\{1\}, V_{12}=\{2,3\}$ and $V_{13}=\{4\}$ with $c^{H_{1}}\left(V_{11}\right)=1, c^{H_{1}}\left(V_{12}\right)=3$ and $c^{H_{1}}\left(V_{13}\right)=2$.

2. $H_{2}$ is defined over $\{1,2,3,4,5\}$ and partitions this set into the three subsets $V_{21}=\{1,4\}, V_{22}=$ $\{3,5\}$ and $V_{23}=\{2\}$ with $c^{H_{2}}\left(V_{21}\right)=2, c^{H_{2}}\left(V_{22}\right)=4$ and $c^{H_{2}}\left(V_{23}\right)=2$.

In this case, the base numbers of $H_{1}$ and $H_{2}$ are 6 and 8 , respectively. Both can be regarded as histogramms over $\{1,2,3,4,5\}$ and their revised, scaled values are

1. $c_{s}^{H_{1}}\left(V_{11}\right)=4, c_{s}^{H_{1}}\left(V_{12}\right)=12, c_{s}^{H_{1}}\left(V_{13}\right)=8, c_{s}^{H_{1}}(\{5\})=0$.

2. $c_{s}^{H_{2}}\left(V_{21}\right)=6, c_{s}^{H_{2}}\left(V_{22}\right)=12$ and $c_{s}^{H_{2}}\left(V_{23}\right)=6$.

Any two histograms that are to be compared will thus be assumed to be over the same set, $V$, and both to have base number, $n$. Let $H_{1}, H_{2}$ denote two such histograms.

If two histograms, $H_{1}$ and $H_{2}$ are defined over the same partition of $V$ into singleton sets, $V=$ $\left\{v_{1}\right\} \cup\left\{v_{2}\right\} \ldots \cup\left\{v_{m}\right\}$ then the obvious metric to use to compare $H_{1}$ and $H_{2}$ is 


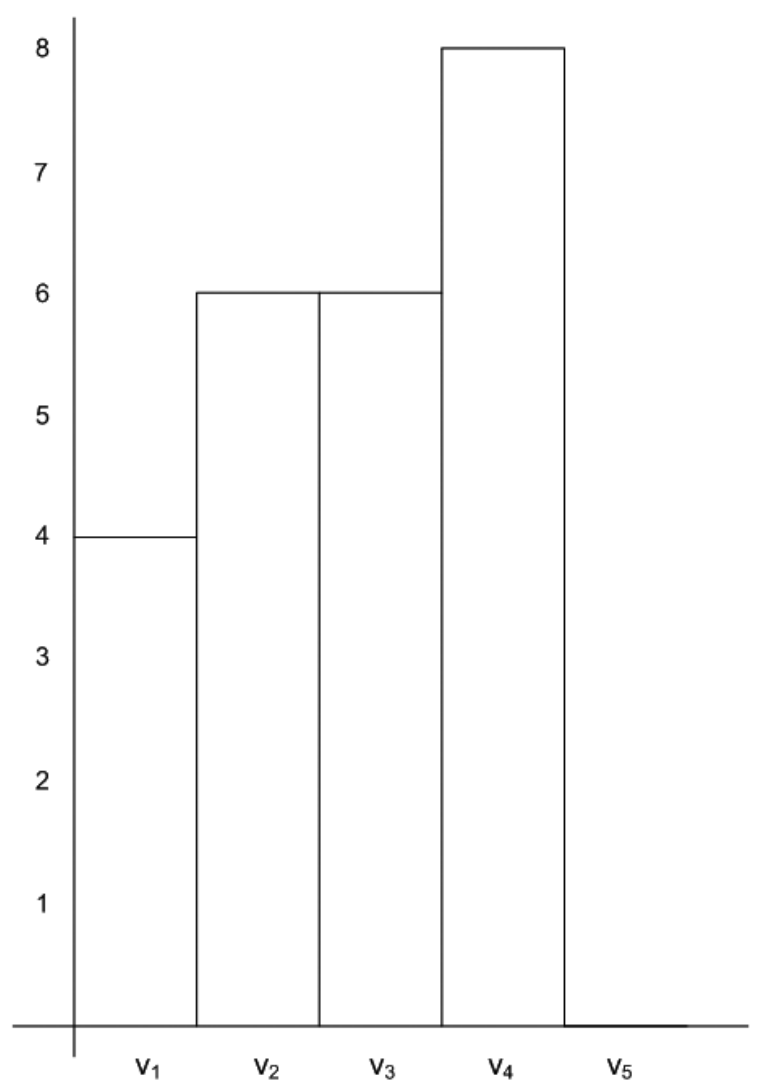

Fig. 6. Derived Representation of (scaled) $H_{1}$.

$$
\delta\left(H_{1}, H_{2}\right)=\sum_{i=1}^{m}\left|c^{H_{1}}\left(v_{i}\right)-c^{H_{2}}\left(v_{i}\right)\right| .
$$

However, when the histograms use different partitions, the metric is not so immediate but is an obvious generalisation. With respect to a histogram, $H$, over $V$, each $v \in V$ can be assigned a derived count value

$$
d^{H}(v)=\frac{c^{H}\left(V_{v}^{H}\right)}{\left|V_{v}^{H}\right|},
$$

where $V_{v}^{H}$ is the set in the partition of $H$ containing $v$. Then the following is clear.

Corollary 5 One metric to compare $H_{1}$ and $H_{2}$ is simply

$$
\delta_{1}\left(H_{1}, H_{2}\right)=\sum_{i=1}^{m}\left|d^{H_{1}}\left(v_{i}\right)-d^{H_{2}}\left(v_{i}\right)\right| .
$$

The two histograms, $H_{1}$ and $H_{2}$, can both be presented diagrammatically in $R^{2}$ where they both have an identically labelled $\mathrm{x}$-axis, which will be some enumeration of $V, v_{1}, v_{2}, \ldots, v_{m}$. The derived representation of histogram $H_{i}(i=1,2)$ is constructed as follows. For each label, $v_{j}$, the rectangle 


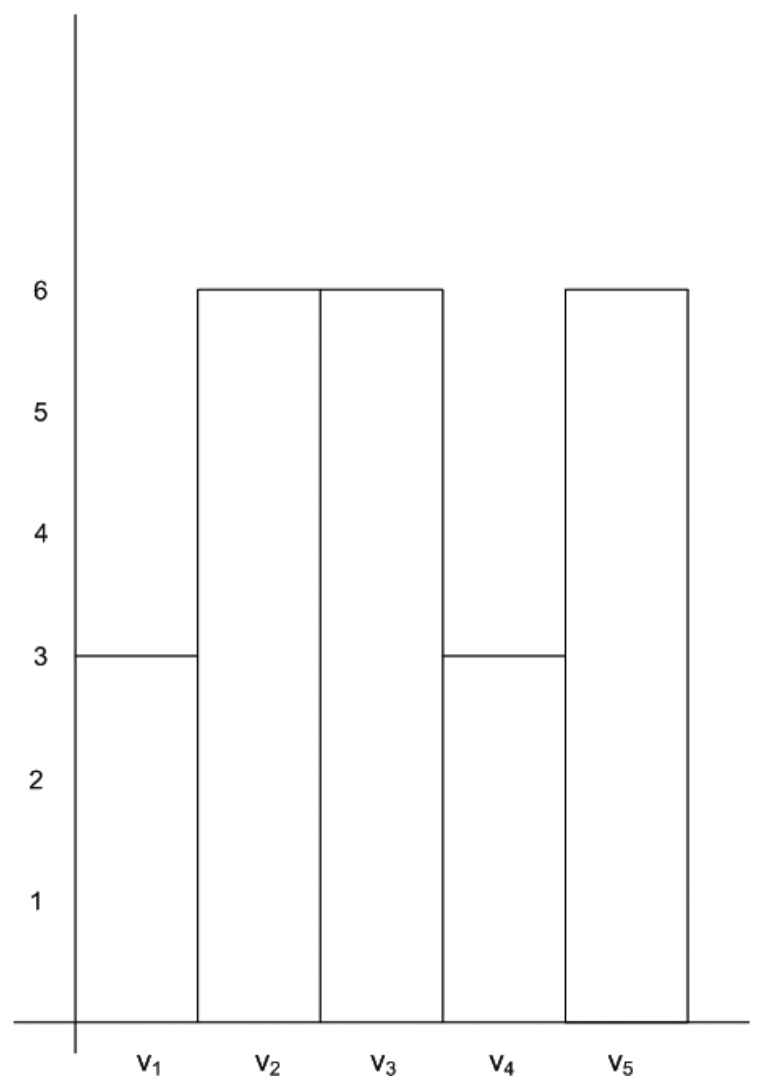

Fig. 7. Derived Representation of (scaled) $\mathrm{H}_{2}$.

$[j-1, j] \times\left[0, d_{i}^{H}\left(v_{j}\right)\right]$ is drawn. Thus if $H_{1}$ and $H_{2}$ are the scaled histograms above, their derived representations are as in Figs 6 and 7. Note that the representation necessarily has area $n$.

The above metric then corresponds to the first metric that can be deduced using proposition 1 from the area measure applied to the two histograms viewed as regions of $[0, m] \times[0, n]$. A second metric then follows from Proposition 2.

\section{Corollary 6}

$$
\begin{aligned}
\delta_{2}\left(H_{1}, H_{2}\right) & =\frac{\sum_{i=1}^{m}\left|d^{H_{1}}\left(v_{i}\right)-d^{H_{2}}\left(v_{i}\right)\right|}{\sum_{i=1}^{m} \max \left(d^{H_{1}}\left(v_{i}\right), d^{H_{2}}\left(v_{i}\right)\right)} \\
& =1-\frac{\sum_{i=1}^{m} \min \left(d^{H_{1}}\left(v_{i}\right), d^{H_{2}}\left(v_{i}\right)\right)}{\sum_{i=1}^{m} \max \left(d^{H_{1}}\left(v_{i}\right), d^{H_{2}}\left(v_{i}\right)\right)}
\end{aligned}
$$

is a metric. Proof:

$\sum_{i=1}^{m}\left|d^{H_{1}}\left(v_{i}\right)-d^{H_{2}}\left(v_{i}\right)\right|$ is the area of the symmetric difference of the two regions defined by $H_{1}$ and $H_{2}$ and is equal to $\sum_{i=1}^{m} \max \left(d^{H_{1}}\left(v_{i}\right), d^{H_{2}}\left(v_{i}\right)\right)-\sum_{i=1}^{m} \min \left(d^{H_{1}}\left(v_{i}\right), d^{H_{2}}\left(v_{i}\right)\right)$. $\sum_{i=1}^{m} \max \left(d^{H_{1}}\left(v_{i}\right), d^{H_{2}}\left(v_{i}\right)\right)$ is the area of the union of these two regions. 
For our example histograms, the first metric provides a distance value of

$$
|4-3|+|6-6|+|6-6|+|8-3|+|6-0|=12
$$

and the second normalises this as

$$
\frac{12}{4+6+6+8+6}=\frac{4}{10} \text {. }
$$

Of course, it may not have been wise to share out the count of the number of occurrences of elements of a partition equally between the elements in that partition as was done with $d^{H}$. Since $d^{H}(v)$ may not be integer valued, there may be no possible database of $n$ elements that could give rise to such a distribution. Also, given two histograms with different partitions but constructed from the same database, the above metrics constructed from $d_{1}^{H}$ and $d_{2}^{H}$ are quite unlikely to measure $H_{1}$ and $H_{2}$ as being distance zero apart.

One might argue that a more reasonable distance measure is to use alternative derived functions $e^{H_{1}}, e^{H_{2}}$, which are both integer valued and are such that

1. If the partition of $H_{1}$ is $V_{11}, V_{12}, \ldots, V_{1 k_{1}}$ then

$$
\sum_{v \in V_{1 j}} e^{H_{1}}(v)=c^{H_{1}}\left(V_{1 j}\right) \text { for all } 1 \leqslant j \leqslant k_{1} .
$$

2. If the partition of $H_{2}$ is $V_{21}, V_{22}, \ldots, V_{2 k_{2}}$ then

$$
\sum_{v \in V_{2 j}} e^{H_{2}}(v)=c^{H_{2}}\left(V_{2 j}\right) \text { for all } 1 \leqslant j \leqslant k_{2} .
$$

3. Subject to the above,

$$
\delta_{\min }\left(H_{1}, H_{2}\right)=\sum_{i=1}^{m}\left|e^{H_{1}}\left(v_{i}\right)-e^{H_{2}}\left(v_{i}\right)\right|
$$

is minimised.

Note, such a distance measure may not itself be a metric since it may not satisfy the triangle inequality. However, if two histograms are constructed from the same database, they will necessarily be distance zero apart as measured by $\delta_{\min }$. This distance measure can be computed using a maximum flow algorithm. A network is constructed as follows.

1. There is a source node labelled, $S$, and from this node, directed edges go to nodes labelled, $V_{11}, V_{12}, \ldots, V_{1 k_{1}}$, where the arc from $S$ to $V_{1 j}$ has capacity $c^{H_{1}}\left(V_{1 j}\right)$ for all $1 \leqslant j \leqslant k_{1}$.

2. There is a node labelled with each $v \in V$ and, for each node labelled $V_{1 j}, 1 \leqslant j \leqslant k_{1}$, there are directed edges to each $v \in V_{1 j}$; these edges all have capacity $c^{H_{1}}\left(V_{1 j}\right)$.

3. There are nodes labelled, $V_{21}, V_{22}, \ldots, V_{2 k_{2}}$ and, for each $V_{2 j}, 1 \leqslant j \leqslant k_{2}$, there are directed edges from each $v \in V_{2 j}$; these edges all have capacity $c^{H_{2}}\left(V_{2 j}\right)$.

4. There is a sink node labelled $T$, and directed edges go to the node $T$ from nodes labelled, $V_{21}, V_{22}, \ldots, V_{2 k_{2}}$, where the arc from $V_{2 j}$ to $T$ has capacity $c^{H_{2}}\left(V_{2 j}\right)$ for all $1 \leqslant j \leqslant k_{2}$.

As a simple example, consider two histograms $H_{1}$ and $H_{2}$, where

1. $V_{11}=\{a, b\}, V_{12}=\{c, e\}, V_{13}=\{d\}$, 


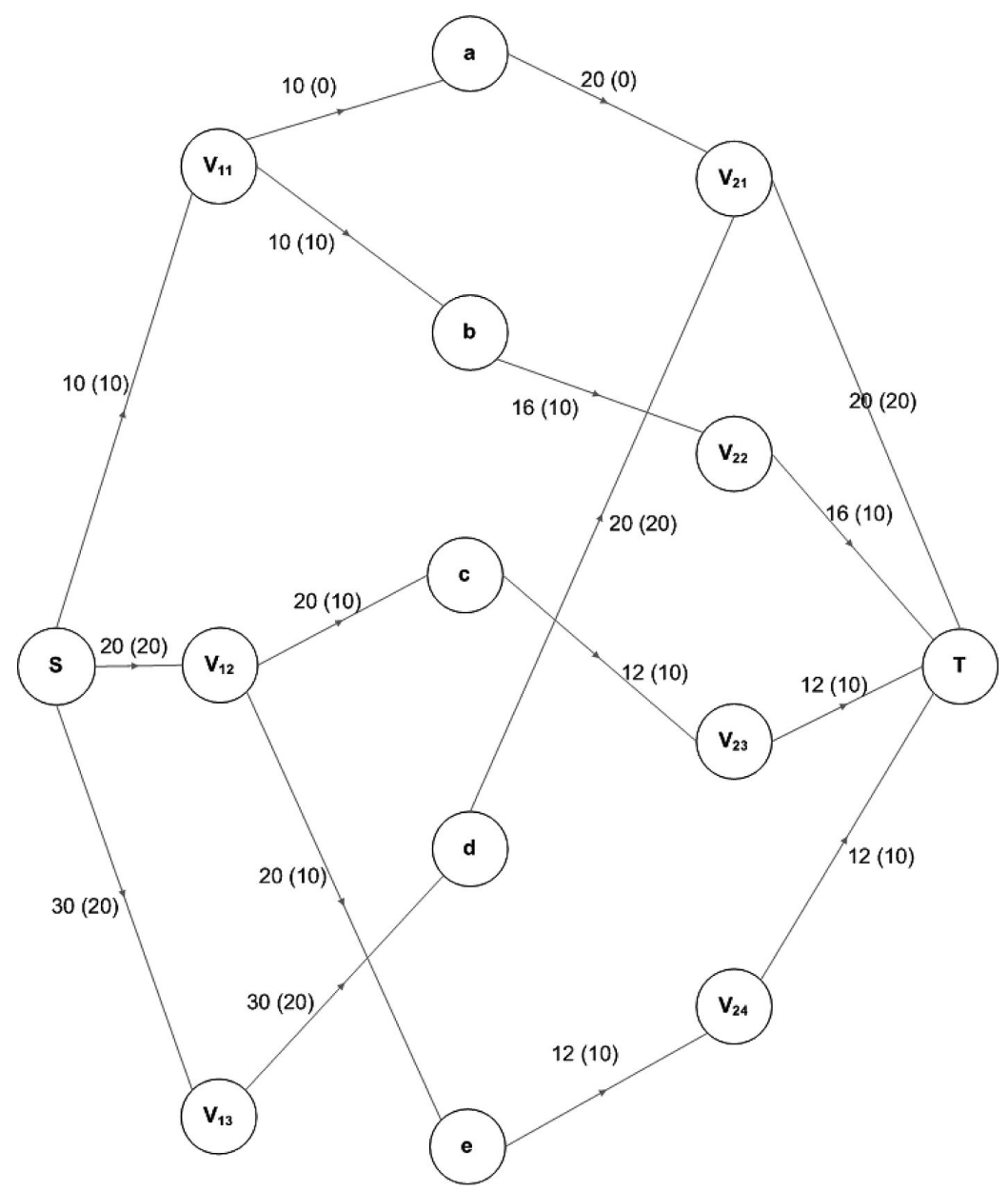

Fig. 8. Constructed network.

2. $V_{21}=\{a, d\}, V_{22}=\{b\}, V_{23}=\{c\}, V_{24}=\{e\}$,

3. $c^{H_{1}}\left(V_{11}\right)=10, c^{H_{1}}\left(V_{12}\right)=20, c^{H_{1}}\left(V_{13}\right)=30$, and

4. $c^{H_{2}}\left(V_{21}\right)=20, c^{H_{2}}\left(V_{22}\right)=16, c^{H_{2}}\left(V_{23}\right)=c^{H_{2}}\left(V_{24}\right)=12$.

The network constructed is then as in Fig. 8.

Let $F$ denote the maximum flow that can be put through such a network for arbitrary $H_{1}, H_{2}$ from the source node to the sink node. This can be computed in $O\left(\mathrm{~m}^{2}\right)$ time using the well known Ford-Fulkerson maximum flow algorithm [18]. For the example of Fig. 8, $\mathrm{F}$ is 50. One possible maximum flow is given in parentheses alongside the arcs in Fig. 8 and it can be seen that this is a maximum flow since $\left\{S, V_{13}, d\right\}$ are separated from the remaining nodes by edges that are saturated.

Theorem 3. $\delta_{\min }\left(H_{1}, H_{2}\right)=2(n-F)$.

Proof:

Consider the maximum flow, $F$ and let the flow through the node labelled $v$ be $f(v)$. For each $V_{1 j}, 1 \leqslant j \leqslant k_{1}$, if $\sum_{v \in V_{1 j}} f(v)=c^{H_{1}}\left(V_{1 j}\right)$ then set $g^{H_{1}}(v)=f(v)$ for all $v \in V_{1 j}$. Otherwise 
$\sum_{v \in V_{1 j}} f(v)<c^{H_{1}}\left(V_{1 j}\right.$ and then select an arbitrary element, $a_{1 j} \in V_{1 j}$, and assign $g^{H_{1}}\left(a_{1 j}\right)=$ $f\left(a_{1 j}\right)+c^{H_{1}}\left(V_{1 j}\right)-\sum_{v \in V_{1 j}} f(v)>f\left(a_{1 j}\right.$, whilst setting $g^{H_{1}}(v)=f(v)$ for all $v \in V_{1 j} \backslash\left\{a_{1 j}\right\}$.

Similarly, For each $V_{2 j}, 1 \leqslant j \leqslant k_{2}$, if $\sum_{v \in V_{2 j}} f(v)=c^{H_{2}}\left(V_{2 j}\right)$ then set $g^{H_{2}}(v)=f(v)$ for all $v \in V_{2 j}$; otherwise select an arbitrary element, $a_{2 j} \in V_{2 j}$ and assign $g^{H_{2}}\left(a_{2 j}\right)=f\left(a_{2 j}\right)+c^{H_{2}}\left(V_{2 j}\right)-$ $\sum_{v \in V_{2 j}} f(v)$ whilst setting $g^{H_{2}}(v)=f(v)$ for all $v \in V_{2 j} \backslash\left\{a_{2 j}\right\}$. Then,

$$
\sum_{v \in V_{1 j}} g^{H_{1}}(v)=c^{H_{1}}\left(V_{1 j}\right) \text { for all } 1 \leqslant j \leqslant k_{1}
$$

and

$$
\sum_{v \in V_{2 j}} g^{H_{2}}(v)=c^{H_{2}}\left(V_{2 j}\right) \text { for all } 1 \leqslant j \leqslant k_{2} .
$$

Note also that for all nodes labelled $v \in V$,

$$
\min \left(g^{H_{1}}(v), g^{H_{2}}(v)\right)=f(v)
$$

since if there is any node where that does not occur, the flow through that node can be increased. For each $V_{1 j}$, if $\sum_{v \in V_{1 j}} f(v)<c^{H_{1}}\left(V_{1 j}\right.$ then there is some arbitrary element of $V_{1 j}$ whose $g^{H_{1}}$ value has been increased to take up the slack, viz. $C^{H_{1}}\left(V_{1 j}\right)-\sum_{v \in V_{1 j}} f(v)$. The total slack across all sets $V_{11}, V_{12}, \ldots, V_{1 k_{1}}$ is $n-F$. This also applies to sets $V_{21}, V_{22}, \ldots, V_{2 k_{2}}$ and hence $\sum_{i=1}^{m} \mid g^{H_{1}}\left(v_{i}\right)-$ $g^{H_{2}}\left(v_{i}\right) \mid=2(n-F)$.

All that is now needed to be established is that $\sum_{i=1}^{m}\left|e^{H_{1}}\left(v_{i}\right)-e^{H_{2}}\left(v_{i}\right)\right|$ is minimised by g. Say it was not and that there is some other choice of functions, $h^{H_{1}}\left(v_{i}\right), h^{H_{2}}\left(v_{i}\right)$ that satify $\sum_{v \in V_{1 j}} h^{H_{1}}(v)=c^{H_{1}}\left(V_{1 j}\right)$ for all $1 \leqslant j \leqslant k_{1}$ and $\sum_{v \in V_{2 j}} h^{H_{2}}(v)=c^{H_{2}}\left(V_{2 j}\right)$ for all $1 \leqslant j \leqslant k_{2}$ but where $\sum_{i=1}^{m}\left|h^{H_{1}}\left(v_{i}\right)-h^{H_{2}}\left(v_{i}\right)\right|<\sum_{i=1}^{m}\left|g^{H_{1}}\left(v_{i}\right)-g^{H_{2}}\left(v_{i}\right)\right|$.

Now, set $f^{\prime}\left(v_{i}\right)=\min \left(h^{H_{1}}\left(v_{i}\right), h^{H_{2}}\left(v_{i}\right)\right)$ and consider a flow, $F^{\prime}$, through the network where $f^{\prime}\left(v_{i}\right)$ passes through node $v_{i}$. This will be a valid flow through the other nodes of the network as well. $\sum_{i=1}^{m}\left|h^{H_{1}}\left(v_{i}\right)-h^{H_{2}}\left(v_{i}\right)\right| \leqslant 2\left(n-F^{\prime}\right)$ so $2 F^{\prime} \geqslant 2 n-\sum_{i=1}^{m}\left|g^{H_{1}}\left(v_{i}\right)-g^{H_{2}}\left(v_{i}\right)\right|>2 n-\sum_{i=1}^{m} \mid e^{H_{1}}\left(v_{i}\right)-$ $e^{H_{2}}\left(v_{i}\right) \mid=2 F$ and this is a contradiction since $\mathrm{F}$ is a maximum flow. The theorem is thus established.

Returning now to the above simple example. If the derived values, $d$ are used then $\delta\left(H_{1}, H_{2}\right)=$ $|5-10|+|5-16|+|10-12|+|30-10|+|10-12|=40$. However, constructing the flow network of Fig. 8, the maximum flow is found to be 50, comprising (say) a flow through $a$ of 0 , through $b$ of 10 , through $c$ of 10, through $d$ of 20 and through $e$ of 10. Hence, $\delta_{\min }\left(H_{1}, H_{2}\right)=2(60-50)=20$. This could arise if $H_{1}$ was a histogram for a database with 0 occurrences of $a, 10$ occurrences of $b, 10$ occurrences of $c, 30$ occurrences of $d$ and 10 occurrences of $e$ and $H_{2}$ was a histogram for a database with 0 occurrences of $a, 16$ occurrences of $b, 12$ occurrences of $c, 20$ occurrences of $d$ and 12 occurrences of $e$.

\subsubsection{Histograms on ordinal sets}

If a histogram, $H$, is over an ordinal field, $\mathrm{F}$, with values in a finite ordered set, $V$, then there is an injective ranking function $\rho: V \rightarrow R^{+}$. A histogram is then based on a partitioning of $V$ into subsets $V_{1}, V_{2}, \ldots, V_{k}$ for some $k>1$. In the case where $V$ is ordinal, $v \in V_{i}$ and $w \in V_{j}$ must satisfy $i<j \Leftrightarrow \rho(v)<\rho(w)$. The elements of $V$ are assumed to be ordered by their $\rho$-value, i.e. $v_{i}<v_{j} \Leftrightarrow \rho\left(v_{i}\right)<\rho\left(v_{j}\right)$, and then, for each $1 \leqslant i \leqslant k, V_{i}=\left\{v_{l_{i}}, v_{l_{i}+1}, \ldots, v_{r_{i}}\right\}$, where 
1. $v_{l_{1}}=v_{1}$ and $v_{r_{k}}=v_{m}$,

2. $v_{r_{i}+1}=v_{l_{i+1}}$ for $1 \leqslant i<k$.

A histogram over $V=V_{1} \cup V_{2} \ldots \cup V_{k}$ will then assign a count, $c^{H}\left(V_{i}\right) \in Z_{0}^{+}$for each $1 \leqslant i \leqslant k$, of the number of occurrences of elements in $V_{i}$ that occur in field $\mathrm{F}$ of the database.

As in Subsection 4.5.1, the assumption is made when comparing two histograms, $H_{1}, H_{2}$, on ordinal sets that both histograms have been scaled if necessary so that they both have the same base number, $n$, and are both defined over the same set, $V$.

The fact that $V$ is ordered can be ignored and, if wished, $V$ can be treated as nominal data. Hence the two metrics of Corollaries 5 and 6 can be used on histograms over ordinal sets. However, such metrics do not exploit the ordering; to do so, a cumulative histogram should be constructed. If $H$ is a histogram over an ordinal set, the cumulative histogram, $\bar{H}$, corresponding to $H$ has the same partition $V_{1}, V_{2}, \ldots, V_{k}$ as $H$ but has count

$$
c^{\bar{H}}\left(V_{i}\right)=\sum_{j=1}^{i} c^{H}\left(V_{j}\right) .
$$

For example, in Fig. 9, $H_{1}$ and $H_{2}$ are two histograms on an ordered set $V=\left\{v_{1}, v_{2}, \ldots, v_{6}\right\}$. $H_{1}$ partitions $V$ into $\left\{v_{1}, v_{2}\right\},\left\{v_{3}, v_{4}, v_{5}\right\}$ and $\left\{v_{6}\right\}$. $H_{2}$ partitions $V$ into $\left\{v_{1}, v_{2}, v_{3}\right\},\left\{v_{4}\right\}$ and $\left\{v_{5}, v_{6}\right\}$. $\bar{H}_{1}$ and $\bar{H}_{2}$ are their corresponding cumulative histograms.

If $H$ is a histogram over an ordinal set $V=\left\{v_{1}, v_{2}, \ldots, v_{m}\right\}$ where $V$ is partitioned into $V_{1} \cup V_{2} \ldots \cup V_{k}$ then, as for nominal data, a derived count can be computed for each $v_{i} \in V$,

$$
d^{H}\left(v_{i}\right)=\frac{c^{H}\left(V_{f(i)}\right)}{\left|V_{f(i)}\right|},
$$

where $V_{f(i)}$ is the set in the partition that contains $v_{i}$. The derived cumulative count for $v_{i}, 1 \leqslant i \leqslant m$, is then

$$
d_{c}^{H}\left(v_{i}\right)=\left\{\begin{array}{cr}
\frac{p_{i}}{\left|V_{1}\right|} c^{\bar{H}}\left(V_{1}\right) & \text { if } f(i)=1, \\
c^{\bar{H}}\left(V_{f(i)-1}\right)+\frac{p_{i}}{\left|V_{f(i)}\right|} c^{H}\left(V_{f(i)}\right) & \text { otherwise, }
\end{array}\right.
$$

where $v_{i}$ is the $p_{i}$ th element of $V_{f(i)}$. A simple induction argument can be used to show that the following result holds.

Proposition $3 d_{c}^{H}\left(v_{i}\right)=\sum_{j=1}^{i} d^{H}\left(v_{j}\right)$.

If $H_{1}$ and $H_{2}$ are histograms over an ordinal set, $V$, two new metrics can be deduced by applying Corollaries 5 and 6 to the histograms $H_{1}^{\prime}$ and $H_{2}^{\prime}$, both with partition $\left\{\left\{v_{1}\right\},\left\{v_{2}\right\}, \ldots,\left\{v_{m}\right\}\right\}$ and with counts $d_{c}^{H_{1}}\left(v_{i}\right), d_{c}^{H_{2}}\left(v_{i}\right)$, respectively. This gives the following result.

Corollary 7 If $H_{1}$ and $H_{2}$ are histograms over an ordinal set, $V=\left\{v_{1}, v_{2}, \ldots, v_{m}\right\}$, then the following are metrics:

1.

$$
\delta_{3}\left(H_{1}, H_{2}\right)=\sum_{i=1}^{m}\left|d_{c}^{H_{1}}\left(v_{i}\right)-d_{c}^{H_{2}}\left(v_{i}\right)\right|
$$



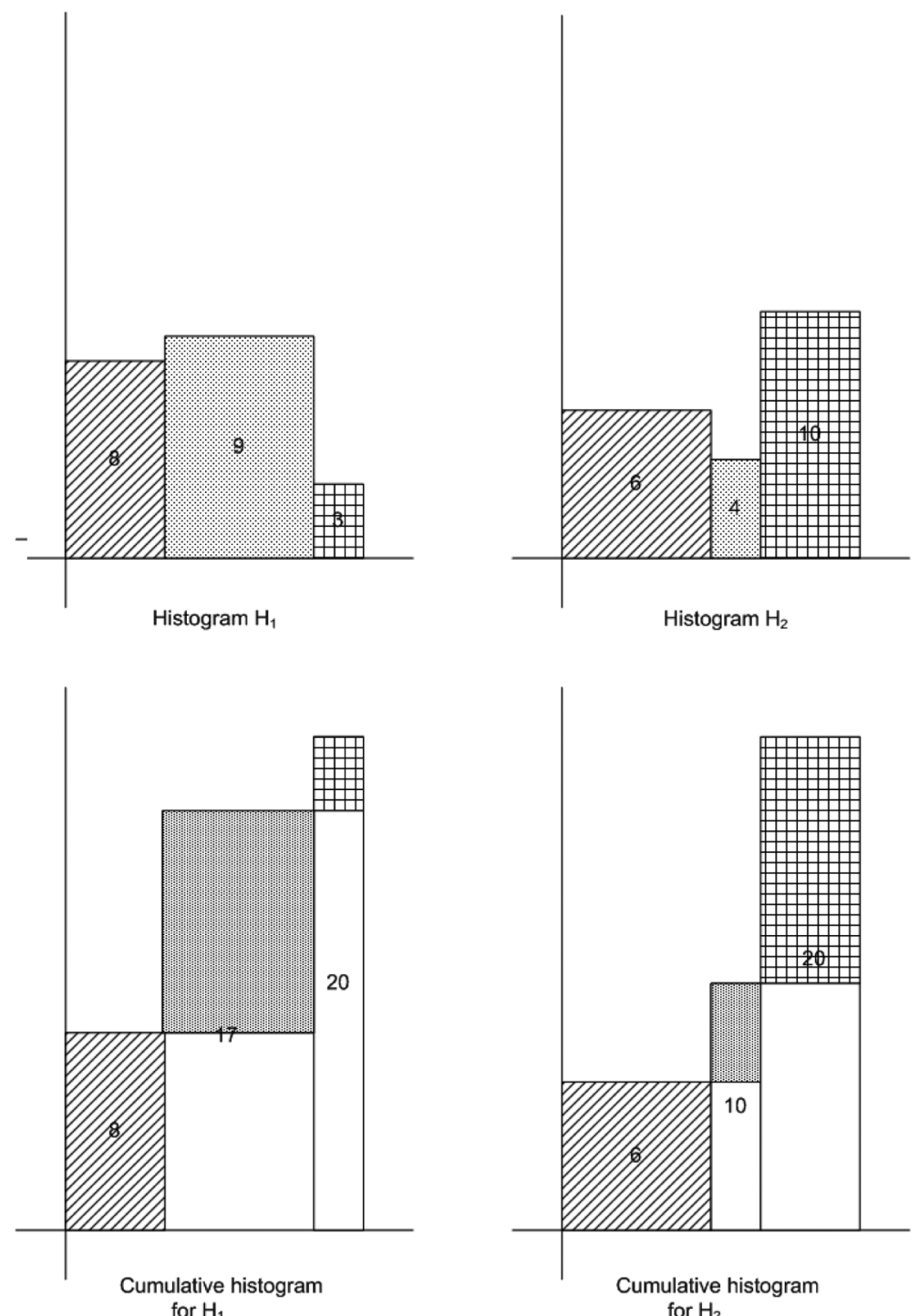

Fig. 9. Two histograms and their correponding cumulative histograms.

$$
=\sum_{i=1}^{m}\left|\sum_{j=1}^{i} d^{H_{1}}\left(v_{j}\right)-\sum_{j=1}^{i} d^{H_{2}}\left(v_{j}\right)\right| .
$$

2.

$$
\begin{aligned}
\delta_{4}\left(H_{1}, H_{2}\right) & =1-\frac{\sum_{i=1}^{m} \min \left(d_{c}^{H_{1}}\left(v_{i}\right), d_{c}^{H_{2}}\left(v_{i}\right)\right)}{\sum_{i=1}^{m} \max \left(d_{c}^{H_{1}}\left(v_{i}\right), d_{c}^{H_{2}}\left(v_{i}\right)\right)} \\
& =1-\frac{\sum_{i=1}^{m} \min \left(\sum_{j=1}^{i} d^{H_{1}}\left(v_{j}\right), \sum_{j=1}^{i} d^{H_{2}}\left(v_{j}\right)\right)}{\sum_{i=1}^{m} \max \left(\sum_{j=1}^{i} d^{H_{1}}\left(v_{j}\right), \sum_{j=1}^{i} d^{H_{2}}\left(v_{j}\right)\right)} .
\end{aligned}
$$


As an example, consider the two histograms, $H_{1}$ and $H_{2}$ of Fig. 9. The derived cumulative counts for $H_{1}$ for the 6 elements $v_{1}, v_{2}, v_{3}, v_{4}, v_{5}$ and $v_{6}$ are $4,8,11,14,17,20$, respectively and for $H_{2}$ they are 2 , 4, 6, 10, 15, 20. Hence

$$
\delta_{3}\left(H_{1}, H_{2}\right)=2+4+5+4+2+0=17
$$

and

$$
\delta_{4}\left(H_{1}, H_{2}\right)=1-\frac{2+4+6+10+15+20}{4+8+11+14+17+20}=\frac{17}{74} .
$$

\subsubsection{Histograms on intervals of the real line}

If a field, F, is real-valued with values in the range $I=(a, b]$, a histogram, $H$, over $I$ then comprises

1. a partition of the interval $I$ into subintervals $I_{1}=\left(l_{1}, r_{1}\right], I_{2}=\left(l_{2}, r_{2}\right], \ldots, I_{k}=\left(l_{k}, r_{k}\right]$ where $l_{1}=a, r_{k}=b$ and $r_{i}=l_{i+1}$ for $1 \leqslant i \leqslant m-1$,

2. for each interval, $I_{i}, 1 \leqslant i \leqslant k$, a count $c^{H}\left(I_{i}\right) \in Z_{0}^{+}$of the number of occurrences of elements of F that lie in $I_{i}$.

Consider two interval histograms, $H_{1}$ over $(a, b]$, and $H_{2}$ over $(c, d]$. Both can be regarded as acting over the same interval, $(\min (a, c), \max (b, d)]$, by setting

1. $c^{H_{1}}(c, a]=0$ if $c<a$ and $c^{H_{2}}(a, c]=0$ if $a<c$,

2. $c^{H_{1}}(b, d]=0$ if $b<d$ and $c^{H_{2}}(d, b]=0$ if $d<b$.

By applying a scaling function

$$
x \mapsto \frac{x-\min (a, c)}{\max (b, d)-\min (a, c)},
$$

it can then be assumed that both histograms are over $(0,1]$. This will be assumed to have been done for any histograms that are to be compared. Moreover, it will be assumed that the base number, $n$, is also the same.

For any histogram, $H$ over $(0,1]$, the cumulative distribution function associated with $H$ is defined as a continuous line from $(0,0)$ to $(1, n)$ such that,

1. over the segment, $I_{1}=\left(0, r_{1}\right]$, it corresponds to the straight line joining $(0,0)$ to $\left(r_{1}, c^{H}\left(I_{1}\right)\right)$, and

2. for $1<i \leqslant k$ over the segment, $I_{i}=\left(l_{i}, r_{i}\right]$, it corresponds to the straight line joining $\left(r_{i-1}, \sum_{j=1}^{i-1} c^{H}\left(I_{j}\right)\right)$ to $\left(r_{i}, \sum_{j=1}^{i} c^{H}\left(I_{j}\right)\right)$.

The cumulative distribution function associated with $H$ will be denoted by $f_{H}$.

Figure 10 gives two histograms over $(0,1]$ together with their cumulative distibution functions. $H_{1}$ partitions $(0,1]$ into $\left(0, \frac{1}{2}\right]$ and $\left(\frac{1}{2}, 1\right] ; H_{2}$ partitions $(0,1]$ into $\left(0, \frac{1}{3}\right],\left(\frac{1}{3}, \frac{2}{3}\right]$ and $\left(\frac{2}{3}, 1\right] ; c_{H_{1}}\left(0, \frac{1}{2}\right]=10$, $c_{H_{1}}\left(\frac{1}{2}, 1\right]=6, c_{H_{2}}\left(0, \frac{1}{3}\right]=8, c_{H_{2}}\left(\frac{1}{3}, \frac{2}{3}\right]=2, c_{H_{2}}\left(\frac{2}{3}, 1\right]=6$.

Corollary 8 If $H_{1}, H_{2}$ are histograms over the interval $(0,1]$ then the following are metrics

1.

$$
\begin{aligned}
\delta_{1}^{I}\left(H_{1}, H_{2}\right) & =\int_{0}^{1}\left|f_{H_{1}}(x)-f_{H_{2}}(x)\right| \\
& =\int_{0}^{1}\left(\max \left(f_{H_{1}}(x), f_{H_{2}}(x)\right)-\min \left(f_{H_{1}}(x), f_{H_{2}}(x)\right)\right)
\end{aligned}
$$



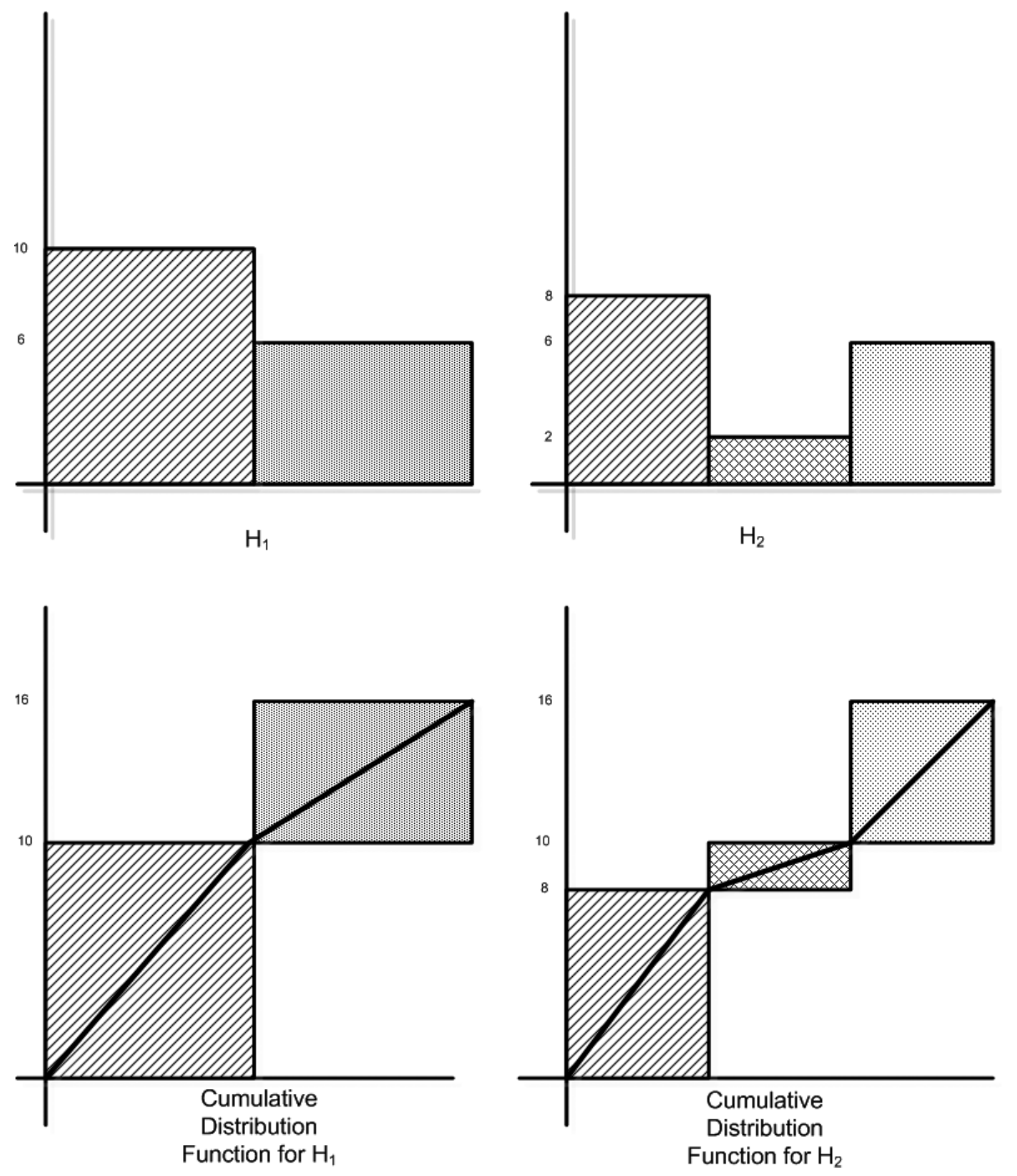

Fig. 10. Two histograms over $(0,1]$.

2.

$$
\delta_{2}^{I}\left(H_{1}, H_{2}\right)=1-\frac{\int_{0}^{1} \min \left(f_{H_{1}}(x), f_{H_{2}}(x)\right)}{\int_{0}^{1} \max \left(f_{H_{1}}(x), f_{H_{2}}(x)\right)} .
$$

Proof: Both results follow immediately from Corollary 4. The first of these metrics is known as the Wasserstein metric and is the usual metric for comparing histograms on intervals, see, e.g. [5,14].

To compute $\delta_{1}^{I}\left(H_{1}, H_{2}\right)$, the points of intersections of $f_{H_{1}}(x)$ and $f_{H_{2}}(x)$ need to be found and then the integral is simply the sum of the differences of areas of trapezia. For example, referring to Fig. 10, the functions $f_{H_{1}}(x)$ and $f_{H_{2}}(x)$ are superimposed in Fig. 11. These two lines only intersect at a single point in $(0,1]$ other than $(1,1)$, i.e. the point of intersection of the line joining $(0,0)$ with $\left(\frac{1}{2}, 10\right)$ with the line joining $\left(\frac{1}{3}, 8\right)$ and $\left(\frac{2}{3}, 10\right)$, viz. $\left(\frac{2}{7}, \frac{4}{7}\right)$. The Wasserstein metric in this case can be computed by computing the difference between the areas of two trapezia in each of the five regions shown. In general, 


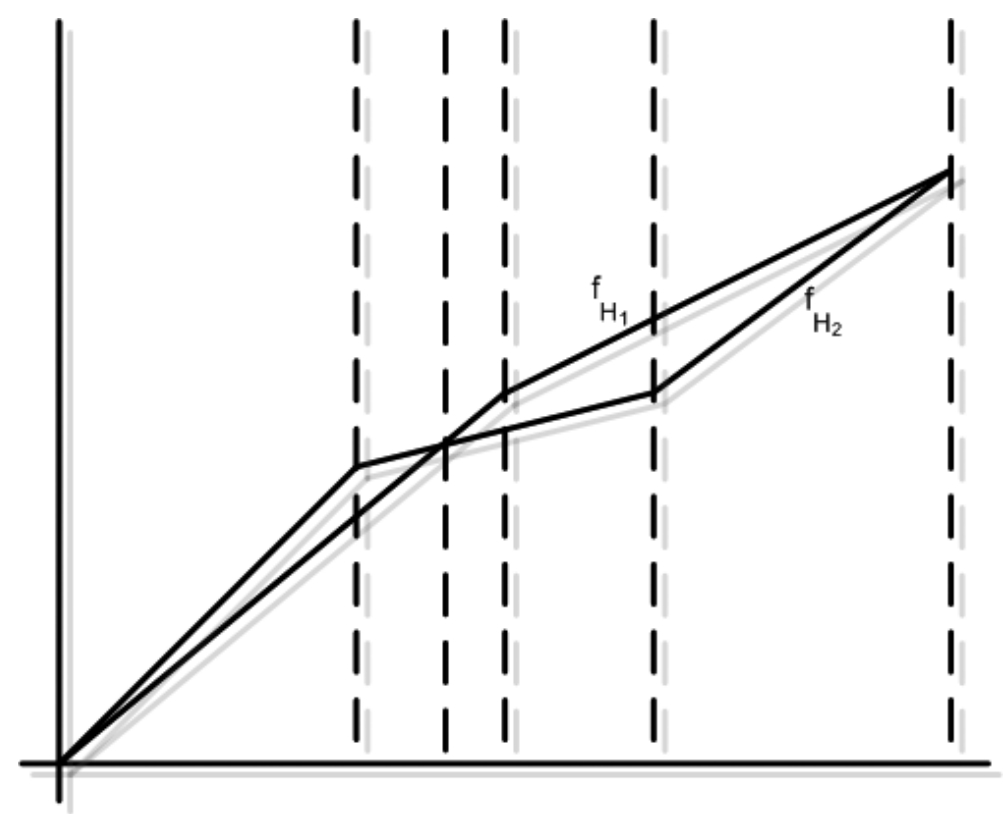

Fig. 11. The intersection of $f_{H_{1}}$ and $f_{H_{2}}$.

if $H_{1}$ has $k_{1}$ intervals and $H_{2}$ has $k_{2}$ intervals, the number of intersection points is at most $\min \left(k_{1}, k_{2}\right)$. The Wasserstein metric can thus be computed in $O\left(k^{3}\right)$ time where $k=\max \left(k_{1}, k_{2}\right)$.

\section{Conclusions and topics for further research}

Two pseudometrics, one of which is normalised, have been shown to exist on an algebra, $(S, \Sigma)$, over which a finitely additive measure, $\mu$, is defined. Provided the measure is strong, both of these pseudometrics have been shown to be full metrics. The first of these metrics is known in the measure theory literature. The normalised version appears to be new.

From these results, metrics or pseudometrics have been deduced for aggregated data in the form of sets, intervals and histograms. Neither of the metrics deduced for nominal sets is new but the second metrics for ordinal sets does appear to be new. The first width metric for intervals is known but again the normalised version has not been found in the literature.

With histograms, it is important to distinguish between histograms over nominal sets, over ordinal sets and over intervals of the reals. The metrics discussed here act on histograms that do not necessarily assume the base set has been partitioned in the same way in both of the two histograms being compared. For histograms over nominal sets, Corollary 6 gives a novel normalised metric. Theorem 3 provides a lower bound on the similarity of two histograms and is new. Finding a similar bound in the ordinal case is an open problem. Of the two metrics for histograms over ordinal sets given in Corollary 7 , the first is the obvious one and, once again, it is the second, normalised metric that appears to be new. This is also the case for histograms over intervals of the real line where the first metric is the Wasserstein metric and the normalised metric appears to be new.

Aggregated data arises following a summarisation process of large databases and may be used as a way of hiding sensitive information on individuals or may be purely part of an analysis process. Important, 
strategic planning decisions can result from the comparison of groups described by aggregated data and a key step in this is to define metrics to measure the difference between aggregated data items relating to two different groups. Normalised metrics have an obvious appeal and, in this paper, a unified theory and notation has been developed from which they can be deduced. Given two groups of individuals, each may have a number of fields, each describing aggregated data. The difference between any two fields can be now be measured but how these differences are best combined to produce a fair and honest, single measure of the difference betwreen the two groups remains a topic for research.

\section{Acknowledgment}

Daniel Sandoval Izarraras provided much of the motivation for this research and discussed some of the underlying ideas with the author.

\section{References}

[1] K.B. Athreya and S.N. Lahiri, Measure Theory and Probability Theory, Springer-Verlag, New York, USA, 2006.

[2] Lynne Billard and Edwin Diday, Symbolic Data Analysis: Conceptual Statistics and Data Mining (Wiley Series in Computational Statistics), John Wiley \& Sons, 2007.

[3] Hans Hermann Bock and E. Diday, Analysis of Symbolic Data: Exploratory Methods for Extracting Statistical Information from Complex Data, Springer-Verlag New York, Inc., Secaucus, NJ, USA, 2000.

[4] Francisco De Carvalho, Paula Brito and Hans-Hermann Bock, Dynamic clustering for interval data based on 12 distance, Comput Stat 21(2) (2006), 231-250.

[5] T.F. Chan, S. Esedoglu and K. Ni, Histogram based segmentation using Wasserstein distances. In Scale Space and Variational Methods in Computer Vision, Springer Lecture Notes in Computer Science 4485, 2007, pages 697-708.

[6] Marie Chavent, Francisco Carvalho, Yves Lechevallier, and Rosanna Verde. New clustering methods for interval data, Computational Statistics 21(2) (June 2006), 211-229.

[7] Francisco de A.T de Carvalho, Renata M.C.R. de Souza, Marie Chavent and Yves Lechevallier, Adaptive hausdorff distances and dynamic clustering of symbolic interval data, Pattern Recogn Lett 27(3) (2006), 167-179.

[8] Renata M.C.R. de Souza and Francisco de A.T. de Carvalho, Clustering of interval data based on city-block distances, Pattern Recognition Letters 25(3) (2004), 353-365.

[9] V. Ganti, R. Ramakrishnan, J. Gehrke, A. Powell and J. French, Clustering large datasets in arbitrary metric spaces, International Conference on Data Engineering, 1999.

[10] Antonio Giusti and Laura Grassini, Cluster analysis of census data using the symbolic data approach, Advances in Data Analysis and Classification 2(2) (2008), 163-176.

[11] K. Chidananda Gowda and T.V. Ravi, Agglomerative clustering of symbolic objects using the concepts of both similarity and dissimilarity, Pattern Recognition Letters 16(6) (1995), 647-652.

[12] D.S. Guru, Bapu B. Kiranagi and P. Nagabhushan, Multivalued type proximity measure and concept of mutual similarity value useful for clustering symbolic patterns, Pattern Recognition Letters 25(10) (2004), 1203-1213.

[13] P.R. Halmos, Measure Theory, van Nostrand, New York, USA, 1968.

[14] A. Irpino, R. Verde and Y. Lechevallier, Dynamic clustering of histograms using Wasserstein metric. In 17th COMPSTAT Symposium of the IASC, 2006.

[15] Antonio Irpino and Rosanna Verde, Dynamic clustering of interval data using a wasserstein-based distance, Pattern Recogn Lett 29(11) (2008), 1648-1658.

[16] P. Jaccard, Étude comparative de la distribution florale dans une portion des Alpes et des Jura, Bulletin de la Société Vaudoise de la Sciences Naturelles 37 (1901), 547-579.

[17] A.K. Jain, M.N. Murty and P.J. Flynn, Data clustering: a review, ACM Comput Surv 31(3) (1999), 264-323.

[18] L.R. Ford, Jr. and D.R. Fulkerson, Maximal flow through a network, Canadian Journal of Mathematics 8 (1956), $399-404$.

[19] Q.H. Nguyen and V.J. Rayward-Smith, Internal quality measures for clustering in metric spaces, International Journal of Business Intelligence and Data Mining 3(1) (2008).

[20] A. Reynolds, G. Richards, B. de laIglesia and V.J. Rayward-Smith, Clustering rules: A comparison of partitioning and hierarchical clustering algorithms, Journal of Mathematical Modelling and Algorithms 5(4) (2006), 475-504. 